\title{
BMJ Open Care needs of dying patients and their family caregivers in hospice and palliative care in mainland China: a meta-synthesis of qualitative and quantitative studies
}

\author{
Shuqin Zhu, ${ }^{1}$ Hanfei Zhu (D) , ${ }^{1}$ Xintong Zhang, ${ }^{2}$ Kouying Liu, ${ }^{1}$ Zumei Chen, ${ }^{3}$ \\ Xiaowen Yang, ${ }^{4}$ Changxian Sun, ${ }^{1,5}$ Weiping Xie, ${ }^{6,7}$ Qin Xu, ${ }^{1}$ Weiying Li, ${ }^{8}$ \\ Dong Pang, ${ }^{9,10}$ Yan Cui (D) , ${ }^{1}$ Hong Wang ${ }^{6,7}$
}

To cite: Zhu S, Zhu H, Zhang $\mathrm{X}$, et al. Care needs of dying patients and their family caregivers in hospice and palliative care in mainland China: a metasynthesis of qualitative and quantitative studies. BMJ Open 2021;11:e051717. doi:10.1136/ bmjopen-2021-051717

- Prepublication history and additional supplemental material for this paper are available online. To view these files, please visit the journal online (http://dx.doi.org/10.1136/ bmjopen-2021-051717).

$\mathrm{SZ}$ and $\mathrm{HZ}$ contributed equally.

Received 29 March 2021 Accepted 12 October 2021

Check for updates

(c) Author(s) (or their employer(s)) 2021. Re-use permitted under CC BY-NC. No commercial re-use. See rights and permissions. Published by BMJ.

For numbered affiliations see end of article.

Correspondence to Dr Hong Wang; hongwang@njmu.edu.cn, Professor Yan Cui; cyan_njmu@163.com and

Professor Qin Xu;

qinxu@njmu.edu.cn

\section{ABSTRACT}

Objective To investigate the care needs of dying patients and their family caregivers in hospice and palliative care in mainland China.

Methods A search for English and Chinese quantitative and qualitative studies was performed using the following English databases: PubMed (Medline), CINAHL and PsycINF0, as well as Chinese databases: SinoMed and CNKI. The records were independently screened by two reviewers and critiqued using Joanna Briggs Institute Critical Appraisal tools. All quantitative data were transformed into qualitative data, which were converted into textual descriptions. Due to the diversity of included studies, a three-step analysis was performed: narrative summary, thematic analysis and presentation of integrated results in a narrative form. The qualitative findings were pooled using the meta-aggregation approach.

Results The literature search identified 2964 papers after removing duplicates, from which 18 were included (9 quantitative and 9 qualitative studies). All studies were conducted in mainland China. Quantitative studies involved cross-sectional surveys, and qualitative studies involved interviews for data collection. Two synthesised results of patients' needs were identified, including needs to be comfortable and experience a good death. Another two synthesised results of family caregivers' needs included needs to care for and improve the quality of life of patients, and to care for themselves well.

Conclusion This study identified that patients and family caregivers have an increasing demand for professional care at the end of life. Professionals, especially nurses, should enact a patients' demand-centred practice to overcome the challenges of organisation, education, emotion and communication to provide high-quality endof-life care.

\section{INTRODUCTION}

The mainland Chinese population is ageing rapidly and has an increasing incidence of some serious diseases, such as malignant tumours. In 2000, the proportion of elderly

\section{STRENGTHS AND LIMITATIONS OF THIS STUDY}

$\Rightarrow$ This is a novel meta-synthesis of qualitative and quantitative studies, conducted in accordance with the Joanna Briggs Institute methodology for mixed methods, to investigate the care needs of dying patients and their family caregivers in hospice and palliative care in mainland China.

$\Rightarrow$ We performed an extensive and systematic literature search to include both qualitative and quantitative data to provide comprehensive insights into the needs of patients and families.

$\Rightarrow$ A limitation of this meta-synthesis is that only studies published in English and Chinese were included, which may cause language bias.

$\Rightarrow$ Some studies were not of high quality but were retained through group discussion, which should be taken into account when interpreting the results of this study.

people (aged 65 years or older) in mainland China was $7 \%$, making the mainland Chinese population an elderly one. In 2019, the proportion of elderly people (aged 65 years or older) in mainland China increased to 167 million $(11.7 \%) .{ }^{1}$ The incidence of malignant tumours has continued to increase annually by $3.9 \%$ over the past 10 years. According to the cancer report published by the National Cancer Center in 2019, the estimated number of new cases of malignant tumours nationwide was 3.804 million, which is an average of more than 10000 people/ day and 7 people $/ \mathrm{min}^{2}{ }^{2}$ With the increasing age and incidence of malignant tumours, there are large potential and actual populations in need of hospice and palliative care; these people need not only assistance with activities of daily living and rehabilitation therapy but also psychological and spiritual 
care. However, the '4-2-1' family structure cannot meet these care needs, thereby compromising the terminal quality of life. ${ }^{3}$ Some patients experience tremendous physical and psychological stress in the terminal stages of life. Three indicators (dependency ratio of the elderly, speed of ageing and the burden of disease) were used to determine the needs for hospice and palliative care, and identify the high demand for hospice and palliative care in mainland China. ${ }^{4}$

People in mainland China do not have access to highquality end-of-life care. According to the Quality of Death Index published by the Economist Intelligence Unit 2015, which ranks the quality of palliative care services, ${ }^{4}$ China ranks the 71st in the world. China's palliative care services are the tenth worst in the world and fourth worst in the Asia Pacific region. In recent years, people have started to pay increasingly more attention towards the quality of death. ${ }^{4}$ To improve the end-stage quality of life, the Chinese government has devised several policies and established 71 pilot cities or districts to improve hospice and palliative care. For example, the State Council and the National Health Commission have published the 'Guiding Opinions on Promoting the Combination of Medical Health and Elderly Services', 5 'the 13th Five-Year Plan for Healthy Ageing ${ }^{6}$ and the 'Notice on the Basic Standards and Management Standards of hospice and palliative Care Centres (Trial Implementation)' ${ }^{7}$

There is a gap between the policies and practice. The government rapidly established wards and service teams. Due to the lack of information regarding the patients' needs during the early stage of practice, nursing services were based on personal experience, which may not have matched the patients' needs. The Chinese culture emphasises on the family as a basis of social relationships. ${ }^{8}$ In China, the family members are often informed of the medical diagnoses before the patients and are required to make medical decisions on behalf of the patients. The patient's own needs (eg, psychological/spiritual needs and need for self-expression) are often ignored. Family caregiving is an important part of Chinese culture. It is regarded caring for the patient as love and reward to the families. ${ }^{8}$ However, family caregiving is associated with a huge burden on the caregivers, particularly on their time, finances, emotions and health; the needs of the caregivers are often neglected while caring for the patient.

It is essential to understand the unmet needs of endstage patients and their family caregivers for developing and improving the hospice and palliative care services and to reduce the gap in practice. ${ }^{8}{ }^{9}$ Previous studies involved a heterogeneous population, were conducted in different areas and had small sample sizes. ${ }^{10}$ Therefore, nurses lack research evidence for useful assessment. This study explored the needs of end-stage patients and their family caregivers by analysing the quantitative and qualitative data from mainland China. The results can guide nurses in accurately assessing the needs and providing evidence based, targeted interventions to patients and their family caregivers.
Prior to this review, there were two systematic reviews on the needs of dying patients and their caregivers, published in March $^{11}$ and February 2021 ${ }^{12}$; however, these reviews used different methods, search sources and study populations compared with the current review. One review ${ }^{11}$ did not exclusively include studies that focus on care needs and included studies conducted in Greater China (including mainland). Because of the differences in the development of hospice care as well as familiarity with and acceptance of hospice and palliative care, results obtained from Hong Kong, Macao and Taiwan may differ from those from the mainland. The other review ${ }^{12}$ used different methods for the theoretical framework, screening, study evaluation, and result synthesis. In this review, we used the Joanna Briggs Institute (JBI) methodology for mixed-methods systematic reviews ${ }^{13}$ and evaluated the studies using standardised critical appraisal instruments. Additionally, we excluded studies with poor quality to provide the best evidence. We followed a convergent integrated approach, in accordance with the JBI methodology for mixed-methods systematic review, which involved integrating the quantised with the qualitative data. Assembled data were categorised and pooled based on similarity in meaning to produce a set of integrated findings. ${ }^{13}$

\section{MATERIALS AND METHODS}

The objective of this study was to quantitatively and qualitatively synthesise scientific evidence on the care needs of dying patients and their families in mainland China. We followed the Preferred Reporting Items for Systematic Reviews and Meta-Analyses (PRISMA) checklist ${ }^{14}$ and the Participants, Phenomena of Interest, Context, Study Types question model to define the inclusion criteria.

\section{Search strategy}

A presearch was performed on PubMed (Medline), Cochrane Library (CDSR) and JBI Evidence Synthesis for previous systematic reviews on this topic to identify the relevant keywords and synonyms. Then, a systematic literature search was performed on PubMed, Cumulative Index of Nursing and Allied Health Literature (CINAHL), PsycINFO, SinoMed and CNKI using Medical Subject Headings (MeSH) and words contained in the titles and abstracts of the relevant articles. To identify the grey literature, the CNKI Database for Thesis and Dissertation was searched. Online supplemental file 1 presents the representative search strategy for PubMed. The search was performed on 9 April 2020 and updated on 27 July 2021, with no restrictions regarding the date of publication.

\section{Inclusion/exclusion criteria}

1. The review included studies that focused on end-oflife hospice patients (eg, expected survival time $<6$ months) and/or their caregivers. 
2. The phenomenon of interest was the care needs of dying patients and their families in mainland China.

3. The context was hospital, home, community, long-term care centre or nursing home. This research focused on quantitative and qualitative studies.

4. The quantitative data designs included, but were not limited to, cross-sectional survey. The methodology of qualitative studies included, but was not limited to, the phenomenology, grounded theory and qualitative description research.

5. Studies were excluded if they were duplicate reports, had unavailable full-text articles, had unavailable data or were published in languages other than English or Chinese.

6. Qualitative studies with appraisal scores below 6/10 (including the 'yes' evaluation items) were excluded. Quantitative studies with appraisal scores below 5/8 (including the 'yes' evaluation items) were evaluated by the research team for inclusion.

\section{Study selection}

All identified citations were collated and uploaded to EndNote (V.X8; Clarivate Analytics, London, UK) to remove duplicate reports. Titles and abstracts were screened by two independent reviewers (SZ and XZ) to assess whether the inclusion criteria were met. Fulltext versions of the selected studies were retrieved and assessed in detail against the inclusion criteria; those that did not meet the inclusion criteria were excluded, and the reasons for exclusion are summarised in the flowchart. Studies selected for inclusion were critically appraised. The search results are presented in a PRISMA flow diagram. Disagreements between the researchers were resolved through discussion or by discussion with a third researcher (KL).

\section{Assessment of methodological quality}

The methodological quality of the included studies was independently evaluated by two reviewers (SZ and XZ) using standardised critical appraisal instruments for quantitative and qualitative studies by JBI. ${ }^{15} 16$ Disagreements between the researchers were resolved through discussion or by discussion with a third researcher (KL). The results of the critical appraisal are reported in narrative and tabulated forms. Data from the studies that met the inclusion criteria were extracted and synthesised.

\section{Data extraction}

Data were independently extracted by two reviewers (SZ and HZ) using a purposefully designed structured data extraction matrix. The extracted data included background information (author names, year of publication, setting and participants). Results of the quantitative (measurement instruments and main findings) and qualitative (phenomena of interest, author-derived themes and descriptions) studies were systematically extracted.

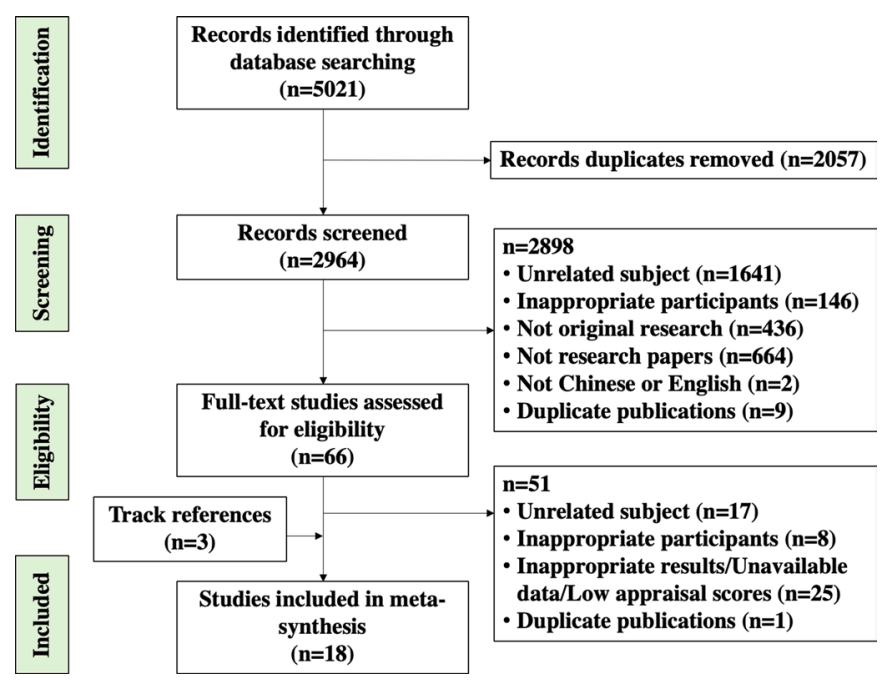

Figure 1 Search results using PRISMA flow chart. PRISMA, Preferred Reporting Items for Systematic Reviews and MetaAnalyses.

\section{Data synthesis}

The quantitative data were transformed into qualitative data, ${ }^{17}$ followed by conversion to textual descriptions for integration with qualitative data. ${ }^{13}$ All qualitative data were analysed in three steps: narrative summary, thematic analysis, and presentation of integrated results in a narrative form. All qualitative findings were pooled using the meta-aggregation approach. ${ }^{13}$ The extracted findings were compared, and similar findings were categorised together. These categories were reviewed and aggregated into synthesised, coherent whole findings. The synthesis process was reviewed by two authors (SZ and HZ) to minimise bias.

\section{Patient and public involvement}

Patients and public were not involved in the review.

\section{RESULTS}

After removal of duplicate reports, we identified 2964 relevant articles. Two researchers simultaneously screened the title and abstract (or executive summary) of the articles. The reference lists of included articles were also checked, which identified three additional relevant studies. Finally, 18 studies were included in this review (9 qualitative and 9 quantitative studies). The screening and selection process for the studies is shown in figure 1.

\section{Study characteristics}

All studies were conducted in mainland China: five in Shanghai, ${ }^{818-21}$ two in Tianjin, ${ }^{22}{ }^{23}$ two in Jiangsu, ${ }^{24}{ }^{25}$ two in Guangdong ${ }^{26} 27$ and one each in Xinjiang, ${ }^{28}$ Hebei, ${ }^{29}$ Beijing ${ }^{30}$ and Hunan. ${ }^{31}$ Wang et $a l^{32}$ Wang et $a l^{33}$ and Liu $e t a l^{34}$ did not specify the study setting in their reports. Eight studies explored the needs of family caregivers of dying patients, while six focused on dying patients and four on both patients and their 
family members. The participants in most studies were cancer patients, except for the study by Wang $e t a l^{32}$ All quantitative studies had a cross-sectional design; most qualitative studies used phenomenological methods. Details of the characteristics of the included studies are listed in tables 1 and 2.

\section{Quality of studies}

All qualitative studies showed congruity among the research question, methodology and result interpretation. Participants and their voices were adequately represented, and the conclusions were based on the data. Liu $e t a l^{34}$ and Wu $e t a l^{24}$ showed unclear congruity between the stated philosophical perspective and research methodology. Moreover, none of the studies included statements regarding the cultural or theoretical perspectives of the researchers or addressed the influences of researchers' perspectives on the study results. Additionally, evidence of ethical approval by the respective body was not presented by any study, except the study by Liu et al. ${ }^{34}$

The methodological quality varied between the nine quantitative studies. The inclusion criteria were clearly stated by all studies, except the study by Liu et $a .^{29}$ The study subjects and setting were described in all studies, except in the study by Zhao et al. ${ }^{30}$ Two studies $^{21} 29$ did not measure the exposure using valid and reliable methods. Objective and standard criteria were not used for measurement of the condition in Miao et al..$^{21}$ Four studies ${ }^{21} 272930$ did not identify the confounding factors. Only Wang $e t a l^{33}$ and Cui $e t a l^{28}$ attempted to control for the confounding factors. The outcomes were measured using valid and reliable methods in all studies, except for three. ${ }^{21} 2729$ All studies used appropriate statistical analyses. The results of our quality assessment are presented in tables 3 and 4 .

\section{Meta-synthesis}

We extracted 52 key findings from 18 included papers, classified as unequivocal (U), credible (C) or not supported (NS), and included in the meta-synthesis. These findings were aggregated into 13 categories on the basis of similar meanings. Based on these 13 categories, 4 findings were synthesised. Figures 2 and 3 show the synthesised findings for patients' needs and family caregivers' needs, respectively. The synthesised findings are described below.

Synthesised finding 1: Patients' need to be comfortable: The patient interviewees stated that their most important care need was reduction in pain and increase in comfort to the maximum extent possible.

\section{Category 1: pain and symptom control}

After experiencing the physical and mental stresses caused by the disease, most patients suffered from pain, dyspnoea or other symptoms that were not effectively controlled at the end of their life. Their primary concern was to relieve their symptoms, especially pain.

By this time, I don't have anything else to ask for, just let me suffer a little less, not too uncomfortable. ${ }^{22}$

\section{Category 2: maintain daily life}

Pain at the end of life has a great impact on sleep. Patients have poor sleep quality, difficulty in falling asleep, easy arousability and fatigue after waking up, which lead to poor mental health. Additionally, incontinence, immobility, malnutrition and loss of sensation lead to pressure ulcers and skin infections. ${ }^{23}$ Oral health is important for patient's taste and nutrition. ${ }^{30}$ Therefore, improved sleep quality and dental and oral care were important for patients to maintain daily life and personal image.

\section{Category 3: emotional and spiritual care}

In the process of fighting cancer, family's company, understanding, support and encouragement provided patients with the tenacity and courage. 'I want to spend more time with my family; I hope my family can spend more time with me. ${ }^{22}$, Such patients need more family support and communication with other patients with similar experiences of the illness, which may increase their confidence in the treatment. ${ }^{22}$ Due to the lack of information regarding the disease at the early stage, some patients are fearful and hopeful about the effectiveness of the treatment, prognosis of the disease and the duration of survival. Therefore, they hoped that the healthcare personnel would give them hints of hope or encouragement, which could make them grateful and excited. ${ }^{18}$ After experiencing substantial psychological changes, such as fear, anxiety and depression, brought about by the disease diagnosis and treatment, patients gradually achieve peace of mind. They wanted to be happy for the rest of their lives and do something meaningful. At the end of the patients' lives, they pondered over the meaning of life and expressed willingness to work for their family and society to realise their self-worth.

Looking back at the first half of my life, I have made some achievements, but now I want to give back to the society... I just do what I can to help people in need financially. ${ }^{18}$

\section{Category 4: professional care resources}

Some patients stated that the strict hospital rules should be adjusted because it is essential for a dying patient to be comfortable. Some patients thought that the nurses were not professional and that they needed to improve their skills to avoid the pain caused by treatments, such as venous transfusions and injections. In addition, the patients had a strong will to be provided with community medical services. ${ }^{20}$ It is reported that patients wish to obtain more illness-related and care-related information. ${ }^{34}$ Some patients also hope that the government 


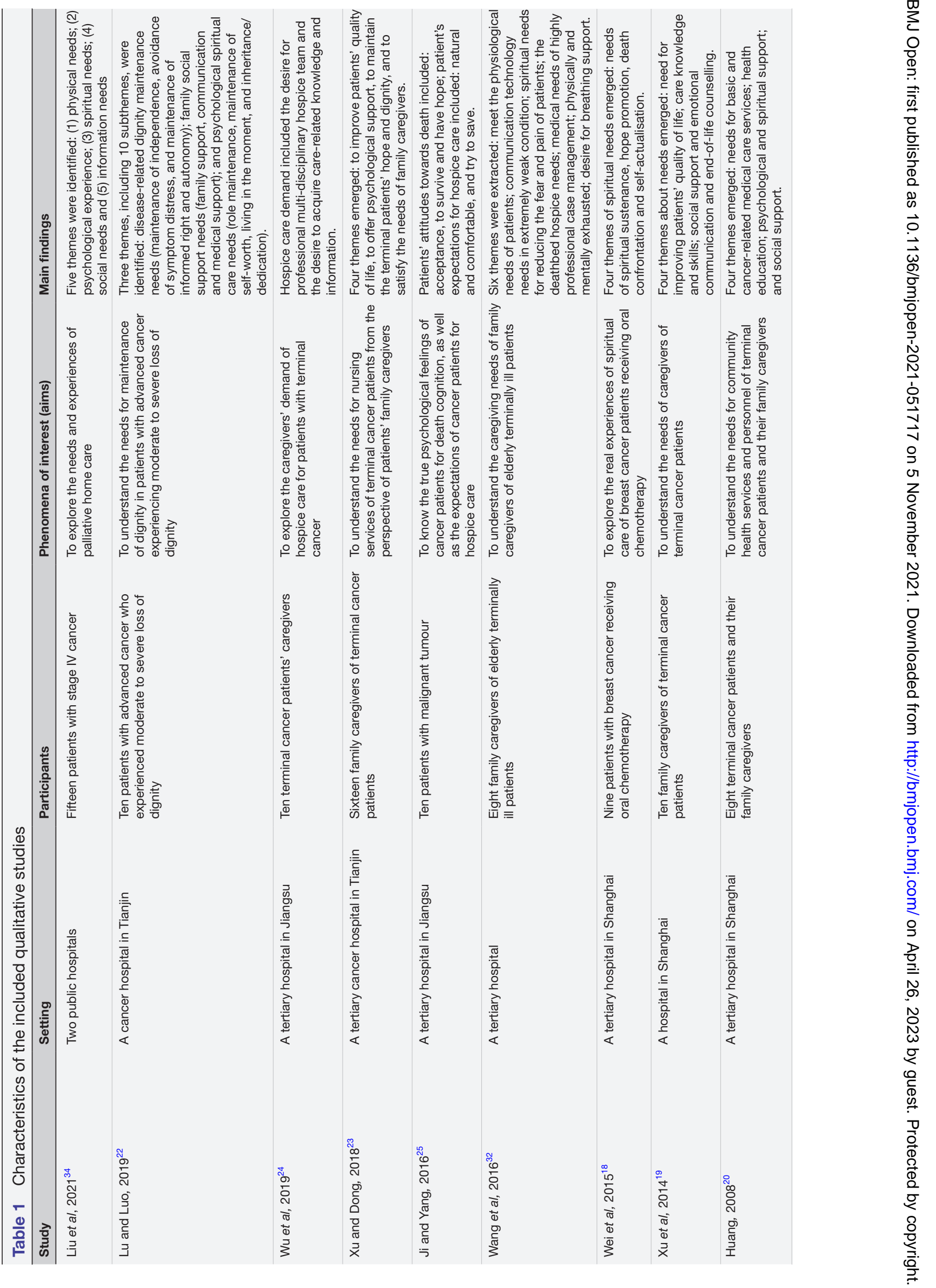




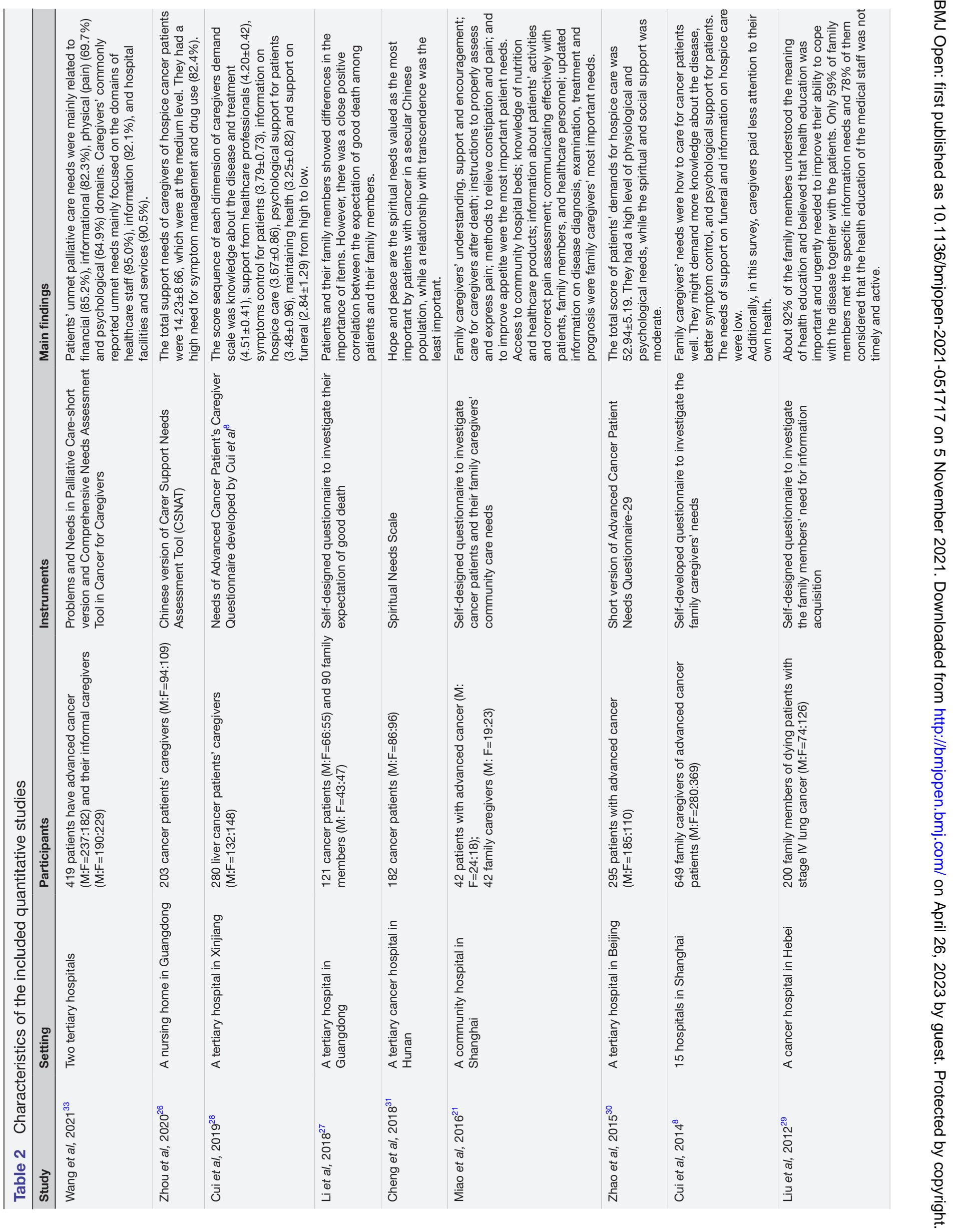


Table 3 Quality assessment of included qualitative studies

\begin{tabular}{|c|c|c|c|c|c|c|c|c|c|c|}
\hline Studies & (1) & (2) & (3) & (4) & (5) & (6) & (7) & (8) & (9) & \\
\hline Liu et al, $2021^{34}$ & UC & Y & Y & Y & Y & $\mathrm{N}$ & $\mathrm{N}$ & Y & Y & \\
\hline Lu and Luo, 2019²2 & Y & Y & Y & $Y$ & Y & $\mathrm{N}$ & $\mathrm{N}$ & Y & UC & $Y$ \\
\hline Wu et al, $2019^{24}$ & UC & Y & $\mathrm{Y}$ & Y & Y & $\mathrm{N}$ & $\mathrm{N}$ & Y & UC & Y \\
\hline Xu and Dong, $2018^{23}$ & Y & $\mathrm{Y}$ & $\mathrm{Y}$ & Y & Y & $\mathrm{N}$ & $\mathrm{N}$ & Y & UC & $\mathrm{Y}$ \\
\hline Ji and Yang, $2016^{25}$ & Y & Y & Y & $Y$ & $\mathrm{Y}$ & $\mathrm{N}$ & $\mathrm{N}$ & Y & UC & Y \\
\hline Wang et al, $2016^{32}$ & Y & $\mathrm{Y}$ & Y & $Y$ & Y & $\mathrm{N}$ & $\mathrm{N}$ & Y & UC & $Y$ \\
\hline Wei et al, $2015^{18}$ & Y & Y & $\mathrm{Y}$ & $\mathrm{Y}$ & $\mathrm{Y}$ & $\mathrm{N}$ & $\mathrm{N}$ & $\mathrm{Y}$ & UC & Y \\
\hline Xu et al, $2014^{19}$ & Y & Y & $\mathrm{Y}$ & Y & $\mathrm{Y}$ & $\mathrm{N}$ & $\mathrm{N}$ & Y & UC & \\
\hline Huang, $2008^{20}$ & Y & Y & $Y$ & $Y$ & Y & $\mathrm{N}$ & $\mathrm{N}$ & $Y$ & UC & $Y$ \\
\hline
\end{tabular}

Y, yes; N, no; UC, unclear

(1) Is there congruity between the stated philosophical perspective and the research methodology?

(2)Is there congruity between the research methodology and the research question or objectives?

(3) Is there congruity between the research methodology and the methods used to collect data?

(4)Is there congruity between the research methodology and the representation and analysis of data?

(5)Is there congruity between the research methodology and the interpretation of results?

(6) Is there a statement locating the researcher culturally or theoretically?

(7)Is the influence of the researcher on the research, and vice-versa, addressed?

(8)Are participants, and their voices, adequately represented? (9) Is the research ethical according to current criteria or, for recent studies, and is there evidence of ethical approval by an appropriate body?

(10) Do the conclusions drawn in the research report flow from the analysis, or interpretation, of the data?

would provide financial support ${ }^{34}$ through the state's medical policy or insurance system.

We are not satisfied with the skill of the nurses, which often causes pain to the patients and aggravates the patient's incompatibility with other treatments. ${ }^{23}$

In the hospital, nurses often give us health education, so the diet and medication compliance is better. At home, I was mentally relaxed, and without professional guidance, my diet and medication were not as regular as they were in the hospital. ${ }^{20}$

Synthesised finding 2: Patients' need of a good death: The end-stage patients hoped that they would get to know of their health condition before they die so that they have the opportunity to say goodbye to their near and dear ones and also complete any pending tasks, before they die peacefully and comfortably in the company of their families at home.

\section{Category 5: maintain autonomy rights}

Many patients expressed the need to maintain autonomy, which included the right of informed consent, rescue, self-esteem and independence, as well as to prolong their lives for as long as possible.
Table 4 Quality assessment of included quantitative studies

\begin{tabular}{|c|c|c|c|c|c|c|c|c|}
\hline Studies & (1) & (2) & (3) & (4) & (5) & (6) & (7) & $\rho$ \\
\hline Wang et al, $2021^{33}$ & Y & Y & $\mathrm{Y}$ & $\mathrm{Y}$ & $\mathrm{Y}$ & $\mathrm{Y}$ & Y & \\
\hline Zhou et al, $2020^{26}$ & $\mathrm{Y}$ & $\mathrm{Y}$ & $\mathrm{Y}$ & $\mathrm{Y}$ & $\mathrm{Y}$ & $\mathrm{N}$ & $\mathrm{Y}$ & $Y$ \\
\hline Cui et al, $2019^{28}$ & $\mathrm{Y}$ & $\mathrm{Y}$ & $\mathrm{Y}$ & $\mathrm{Y}$ & $\mathrm{Y}$ & $\mathrm{Y}$ & Y & Y \\
\hline Li et al, $2018^{27}$ & $\mathrm{Y}$ & Y & $\mathrm{Y}$ & Y & $\mathrm{N}$ & $\mathrm{N}$ & UC & $Y$ \\
\hline Cheng et al, $2018^{31}$ & $\mathrm{Y}$ & $\mathrm{Y}$ & Y & Y & Y & UC & $\mathrm{Y}$ & Y \\
\hline Miao et al, $2016^{21}$ & $\mathrm{Y}$ & $\mathrm{Y}$ & $\mathrm{N}$ & $\mathrm{N}$ & $\mathrm{N}$ & $\mathrm{N}$ & UC & $Y$ \\
\hline Zhao et al, $2015^{30}$ & $\mathrm{Y}$ & $\mathrm{N}$ & $\mathrm{Y}$ & $\mathrm{Y}$ & $\mathrm{N}$ & UC & $\mathrm{Y}$ & $Y$ \\
\hline Cui et al, $2014^{8}$ & $\mathrm{Y}$ & $\mathrm{Y}$ & $\mathrm{Y}$ & Y & Y & NA & Y & $Y$ \\
\hline Liu et al, $2012^{29}$ & NA & Y & $\mathrm{N}$ & Y & $\mathrm{N}$ & $\mathrm{N}$ & $\mathrm{N}$ & \\
\hline \multicolumn{9}{|c|}{$\begin{array}{l}\text { Y, yes; N, no; UC, unclear; NA, not applicable } \\
\text { (1) Were the criteria for inclusion in the sample clearly defined? } \\
\text { (2) Were the study subjects and the setting described in detail? } \\
\text { (3) Was the exposure measured in a valid and reliable way? } \\
\text { (4) Were objective, standard criteria used for measurement of the } \\
\text { condition? } \\
\text { (5) Were confounding factors identified? } \\
\text { (6) Were strategies to deal with confounding factors stated? } \\
\text { (7) Were the outcomes measured in a valid and reliable way? } \\
\text { (8) Was appropriate statistical analysis used? }\end{array}$} \\
\hline
\end{tabular}

In mainland China, many patients were not aware of their disease condition due to concealment by health personnel and family members. However, the patients felt they had the right to be informed to allow them to make decisions for themselves. Some patients insisted on prolonging their lives as long as possible because they believed that one should not give up on life until death. ${ }^{25}$ Some patients also expressed that being able to perform activities of daily life was necessary to maintain self-esteem. The patients believed that if they were unable to maintain their independence, they would become a burden on their families, which would further increase their distress and pain. ${ }^{22}$

If it really comes to the end and there is nothing to do, I have told them (my family members) to give up the treatment. They have to obey my wishes and respect my thoughts. If my family doesn't do this, I will refuse treatment. ${ }^{22}$

If I could not take care of myself, they (my family members) have to respect my feelings and end my life as soon as possible. ${ }^{22}$

\section{Category 6: die peacefully and dignified}

Because of the influence of the traditional Chinese idea of 'falling leaves returning the roots,' most patients preferred to die at home ${ }^{27}$ or at their favourite place. Patients who have experienced the death of a family member preferred to die peacefully and naturally in the company of their loved ones. Many patients expressed that they wanted their rights and personality to be respected at the end of their lives. Some patients also expressed their wish of interpersonal 


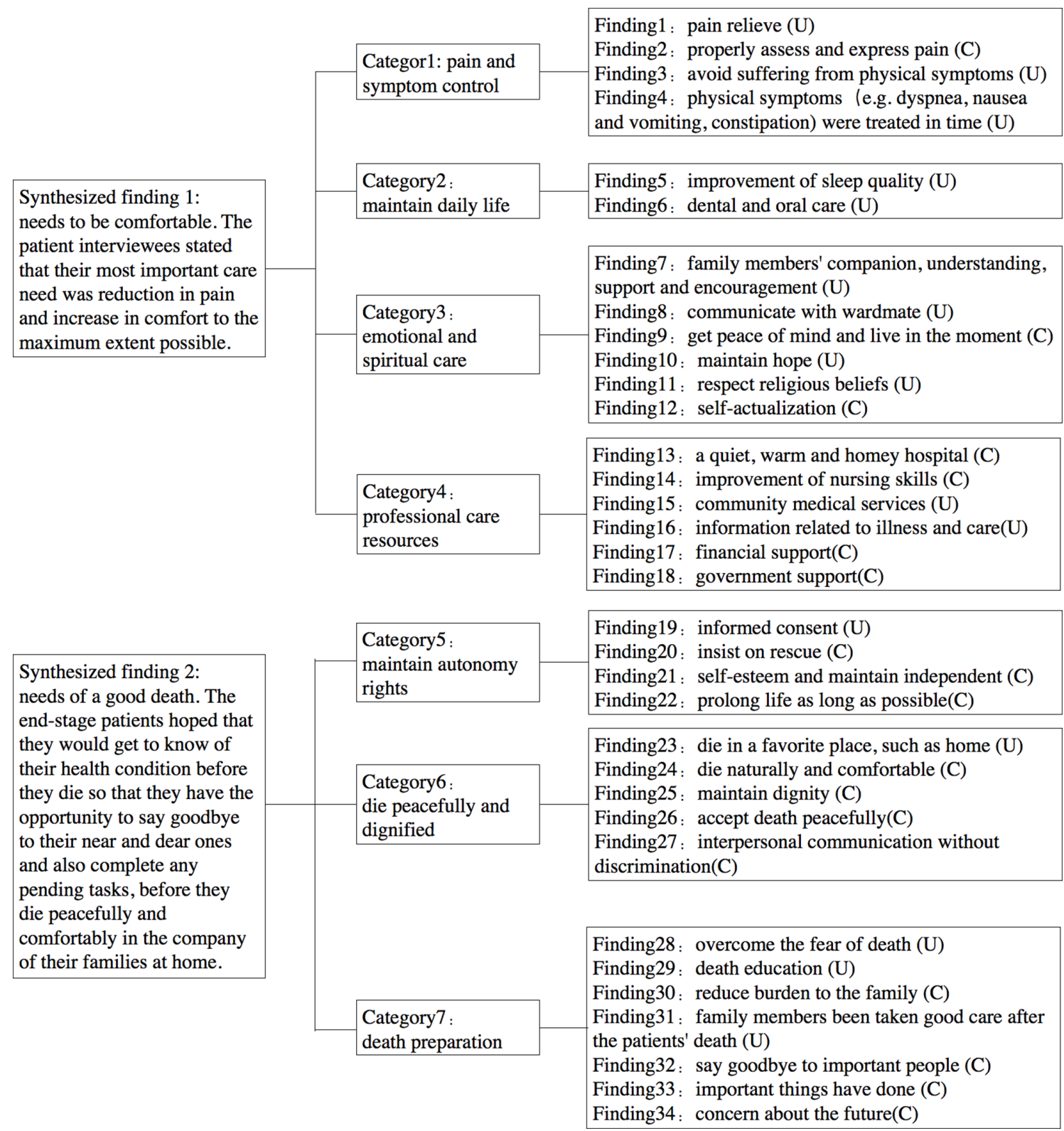

Figure 2 Meta-synthesis findings of the patients' needs.

communication without discrimination and being looked down on by others. ${ }^{34}$

My father also died from cancer. At that time, he was in great pain and didn't want to be treated. However, under the pressure of traditional concept, we kept treating him. He was in such pain, so I just want to leave comfortably when I get to the end, and not receive meaningless therapy. ${ }^{25}$

You see, I have no hair now. I am afraid of going out. If someone comes from home, I have to put on a hood before I open the door. I used to love beauty, so I wanted to leave clean and beautiful. ${ }^{22}$

\section{Category 7: preparation for death}

Most patients expressed their fear of death and concern about their future. However, they were less willing to talk about death and related topics. Death education is often lacking due to a lack of discussion about death. In fact, dying patients desire to overcome their fear of death and expect to be provided with death education from healthcare personnel. However, the lack of discussion about death may be due to the late provision of hospice care and lack of widespread death education. ${ }^{8}$ Many patients cared about their families more than about themselves. They did not want their families to experience psychological or financial burdens. Before death, patients hoped to complete important tasks and say goodbye to important people in their lives. ${ }^{27}$ They also wanted that their family members are taken care of after their death.

I am afraid of death and I hope I could discuss with someone about death. If I can understand and accept it and make arrangements for it, I can at least be less afraid. I will tell my son that mom is always there, in 


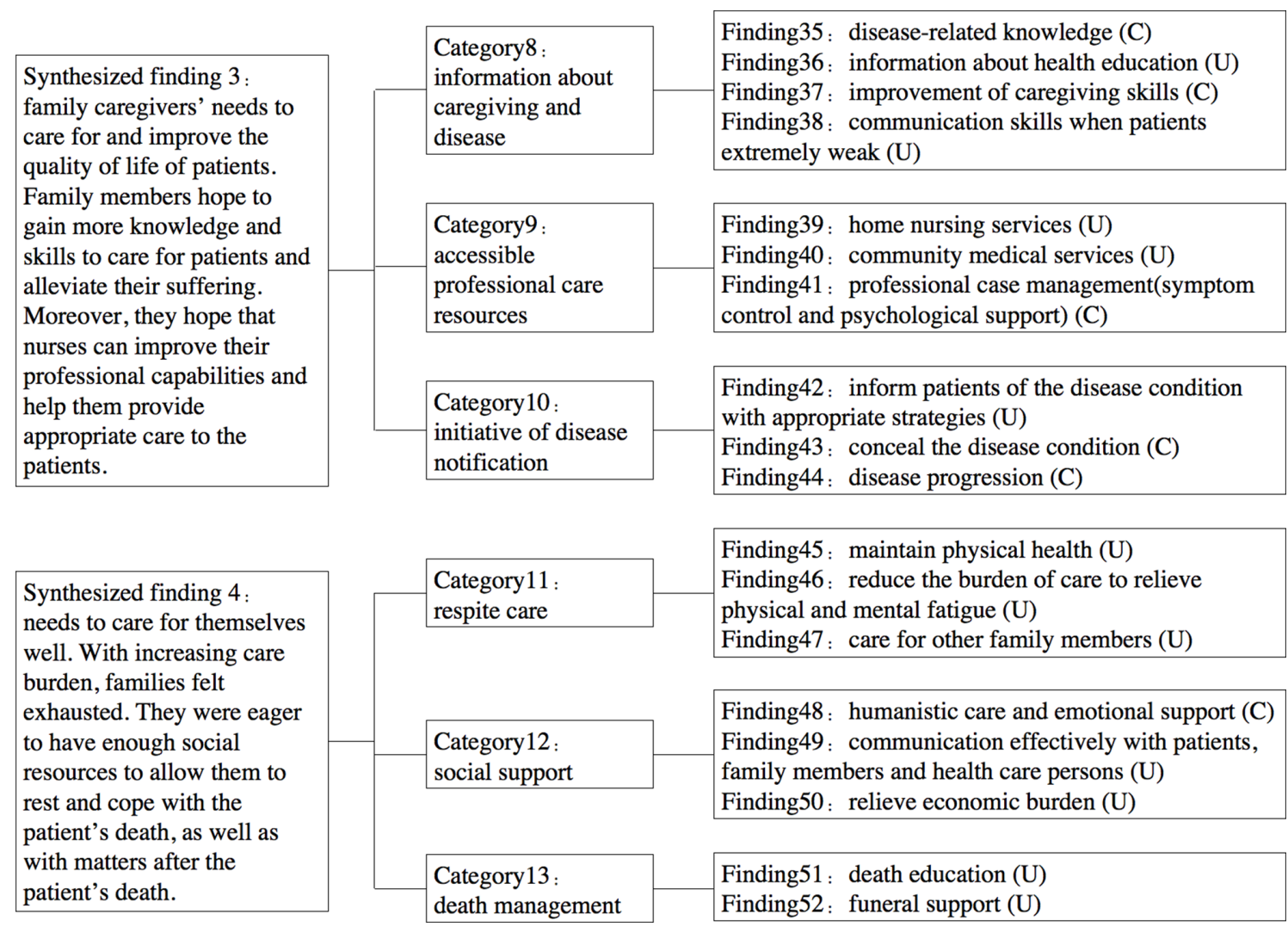

Figure 3 Meta-synthesis findings of the family caregivers' needs.

your heart and love you forever. But there is no such way (death education). ${ }^{18}$

If I can move, I'll do whatever I want and I like, such as fishing. ${ }^{22}$

I am afraid of death and I hope I could discuss with someone about death. If I can understand and accept it and make arrangements for it, I can at least be less afraid. I will tell my son that mom is always there, in your heart and love you forever. But there is no such way (death education). ${ }^{18}$

I am afraid of death and I hope I could discuss with someone about death. If I can understand and accept it and make arrangements for it, I can at least be less afraid. I will tell my son that mom is always there, in your heart and love you forever. But there is no such way (death education). ${ }^{18}$

Synthesised finding 3: Family caregivers' needs to care for and improve the quality of life of patients: Family members hope to gain more knowledge and skills to care for patients and alleviate their suffering. Moreover, they hope that nurses can improve their professional capabilities and help them provide appropriate care to the patients.

\section{Category 8: information about caregiving and disease}

Due to the lack of caregiving knowledge and skills, many family caregivers felt helpless when caring for patients. To better care for patients, family caregivers require information about caregiving and the disease. The caregivers were eager to obtain health education about the disease, drugs, nutrition, rehabilitation and exercise. For patients who were extremely weak and could not express themselves clearly, effective communication between patients and their families was problematic.

Since he got this disease, I often feel very helpless. I don't know what to do, and I really hope that a professional nurse to introduce us to some daily diet and disease knowledge. ${ }^{20}$

\section{Category 9: accessible professional care resources}

Caregivers hoped that the patients would spend their final days at home, but this was difficult to implement due to the lack of home nursing services. Therefore, some caregivers chose community hospitals to provide hospice care for patients. With disease progression, patients' conditions became increasingly complex, and the caregivers desired professional care to assist them in providing comprehensive care for the patients (including symptom control and psychological support).

I hope that there could be more accessible medical resources. If we have any health problem, we could call medical personnel for help. ${ }^{32}$

\section{Category 10: initiative of diagnosis communication}

Many family caregivers believed that patients who were not aware of their diagnosis led better lives and that they would lose hope if informed of the diagnosis. Therefore, 
the families required the healthcare personnel to either not disclose the diagnosis to the patients or to inform them in an appropriate manner. Additionally, many caregivers wanted to be informed regarding the disease progression before the patients, due to which the doctors informed the disease condition to the family members first.

If I told him that the cancer had spread and metastasized, I was afraid that he would collapse at once and live for only a few days. Therefore, we told the nurse to hide the truth and help us to tell this lie. ${ }^{23}$

Synthesised finding 4: Family caregivers' need to care for themselves: With increasing care burden, families felt exhausted. They were eager to have enough social resources to allow them to rest and cope with the patient's death, as well as with matters after the patient's death.

\section{Category 11: respite care}

The caregivers desired appropriate social resources for respite care. If caregivers received assistance, they could have time to care for other family members and relieve their burden.

It would be nice if someone could take care of my wife during the work time, so that I could go to work and don't have to resign. I'm really tired and often get headache. I really hope someone could help me..$^{20}$

\section{Category 12: social support}

In addition to physical fatigue, caregivers also needed to relieve their psychological stress. At the end of a patient's life, the caregivers wanted to communicate effectively with the patient, family members and healthcare personnel because they required emotional support from these people. For many families, cancer treatment was expensive and unaffordable, if they did not have medical insurance. Therefore, they wanted to relieve their economic burden.

Since he (my husband) got this disease, I often have insomnia and worried about his health and my family. I couldn't sleep all night. Sometimes I wish I could have a place to talk to someone. ${ }^{20}$

\section{Category 13: death management}

When the patient is at the terminal stage, some families cannot cope with a patient's death, especially if the family support system is inadequate. Although caregivers did not express their needs for death education, they wanted the medical staff to help them prepare for the patient's death. For families who experience grief, information about funeral support was imperative. Funerals are necessary to improve the feelings of bereavement and reverence. In line with this, many families need funeral support to provide good after-death care to patients.

I was not ready yet. Someone asked me to buy the mourning clothes in advance. I could not accept it and felt upset. At that time, I hoped a nurse to help me. ${ }^{23}$

People's view of life and death is not the same, which is related to the level of education. Some people especially a lower level of education is not open to death, a higher level of education will understand it. We hope that if the nurses know this, they could talk to us in the right way. ${ }^{23}$

\section{DISCUSSION}

The results of this study found that dying patients and family caregivers have a high demand for professional endof-life care, including physical, psychological, social and spiritual care. This is consistent with a previous systematic review that reported that advanced cancer patients and informal caregivers had unmet psychological and physical needs. ${ }^{33}$ The studies included in the current review mainly focused on cancer patients and their family caregivers. The existing studies mainly focused on the healthcare needs of elderly people, particularly in the hospice ward setting, as well as resource acquisition, medical insurance, health services and psychological experience of patients and caregivers. However, such studies did not meet the inclusion criteria for this review. There is a lack of highquality studies on the needs of dying older people, and this review only included one such study. There is also a scarcity of studies on the needs of other patient groups, such as ICU or stroke patients, and none of them met the inclusion criteria of this study. This may be because attention to hospice care began in recent 5 years. Future studies should also include this population.

The primary concern of dying patients was regarding their pain and symptom control, which may be because, as stated by Maslow's theory, the physiological needs should be prioritised. ${ }^{35}$ However, pain receives insufficient attention and is not adequately assessed. It was reported that about $41.3 \%-52.9 \%$ of patients with cancer are dissatisfied with pain management. ${ }^{36}$ Limited use of opioid analgesics and insufficient staff knowledge regarding pain management in mainland China may be responsible for these unmet needs. ${ }^{37}$ Actually, in many countries, the availability of opioids is constrained by legal restrictions, a lack of training and awareness, and social stigma. ${ }^{4}$ These findings indicate that effective strategies are needed to provide professional symptom control for dying patients.

In addition, dying patients not only desire physical comfort but also psychological and spiritual comfort. They wish to achieve peace of mind, live in the moment and maintain hope. Moreover, religious beliefs and selfactualisation are also expressed. The power of the spirit is strong in dying patients, which includes discovering the purpose of life and acquisition of individual inner peace during transcendence. ${ }^{38}$ Some Chinese people with religious beliefs are deeply influenced by Confucianism, Taoism or Buddhism, which creates different concepts of God and religion ${ }^{39}$; however, most Chinese are atheists. 
The connotation of spiritual care and methods to provide it need to be explored further. Patients wish to die peacefully, in a dignified manner and without pain, due to suffering from the disease; some patients hope for positive treatment outcomes because of their desire for life, in accordance with the patients' ambivalence at the end of life. ${ }^{40}$ Thus, healthcare professionals should recognise this need and the ambivalence of patients. The different needs of these patients should be assessed, and individualised interventions should be administered.

Overall, family caregivers wished to care for themselves and the dying patients well. Furthermore, caregivers wanted their beloved to be taken better care of than themselves. Their primary concern was to provide attentive care for patients, ${ }^{8}$ which was hindered by a lack of caregiving knowledge and skills. Professionals could help family caregivers by offering tailored health education to increase their knowledge and skills. The caregivers not only wanted the healthcare professionals to provide information but also professional case management directly for dying patients. ${ }^{32}$ Due to the importance of family and filial piety in China, caregivers focus on the care of their beloved more than their own health; many of them also thought that their self-sacrifices were required and irreplaceable. ${ }^{41}$ Furthermore, many caregivers experienced prolonged grief and high level of stress. ${ }^{42}$ They also felt exhausted emotionally and physically due to the lack of self-care ability of dying patients and heavy caregiver burden. Significant changes have occurred in the family structure in China; the '4-2-1' family structure of the one-child generation is becoming more common, which leads to heavier burden on the spouse and offspring to care for patients. ${ }^{43}$ It is crucial for caregivers to receive professional humanistic care and emotional support. To reduce caregiving burden, respite care was increasingly being demanded. Respite care as an alternative and transient service could provide caregivers with a temporary opportunity to release pressure. ${ }^{44}$ It could also offer caregivers time for socialisation and leisure activities to ease the caregiver burden, adjust their mood, and meet caregivers' demands. Nevertheless, there is a lack of respite care in mainland China, and there are limited human resources (social workers and volunteers) to provide this much-needed service. ${ }^{45}$ There is great demand to develop daycare or respite and intermittent care centres to provide formal and informal help for family caregivers to balance their caregiver role and personal needs.

There are several similarities among patients' and caregivers' needs. Most patients and caregivers reported high demand for home-based hospice care. This is because most patients wished for a good death at home, in accordance with traditional death practices. ${ }^{46}$ Home-based hospice care not only provides patients with various cares at home in their original lifestyle but also reduces medical costs and improves caregivers' anxiety, depression, and other negative emotions. ${ }^{47}$ In the USA, about $55.6 \%$ of palliative care was performed at patients' home. ${ }^{48}$ However, home-based hospice care is still developing in mainland China and is hampered by limited human resources and professional training. ${ }^{49}$ In addition, inadequate salary is another prominent impediment to the delivery of home-based hospice care. At present, there is no policy regarding the implementation of home-based hospice care, such as service mode, charge standard and staff arrangement, which may also hinder the development of hospice care. Some pioneers have made attempts to improve this situation. More studies are required in this area, which will also help the government in formulating policies and developing home-based hospice care to meet the needs of patients and their families.

It is important to consider the differences in patients' and caregivers' needs of informed consent. In mainland China, medical staff and family members often conceal patients' diagnoses. Except for a small number of patients who have medical knowledge and from whom the diagnosis is difficult to conceal, doctors usually do not inform dying patients of their disease condition or progression but communicate with their family members and discuss major issues with them (such as the formulation and implementation of treatments). ${ }^{45}$ Because of the avoidance of death in Chinese traditional culture, it is common for family members to make decisions, instead of patients, to avoid emotional stress to the patient. In essence, this is a type of psychological care with modification according to Chinese characteristics. Because many patients are not aware of their diagnosis, they hope for recovery and do not plan to complete their pending tasks. It may be useful to educate people about palliative care and change their attitudes toward death, specifically regarding avoidance of discussion about death. Zeng et $a \tilde{l}^{0}$ found that it was necessary to evaluate the patient's age, residence, education, occupation, treatment cost and other conditions before informing them of their diagnosis and adopt various strategies accordingly. Additionally, healthcare professionals should guide the family members regarding the appropriate attitude and viewpoint to achieve support and cooperation. Informing the patients regarding their diagnosis is not only conducive to their autonomy rights but also improves the satisfaction of dying patients and their families.

\section{Limitations and future directions}

Although this was a rigorously conducted meta-synthesis, designed using JBI methodology for mixed methods, several limitations were observed. First, only studies published in English or Chinese were included, which may cause language bias. Besides, the participants in most studies had advanced cancer, and there is a lack of information about the needs of other populations, which may cause sampling bias. Although these studies provide important information about the needs of specific subpopulations, lack of research on heterogeneous samples of dying patients restricts our understanding of the needs of the wider population. Therefore, further exploration of dying patients' needs is necessary. Finally, few quantitative studies have used standardised needs assessment 
measures and most studies used self-designed questionnaires. As a result, these studies may not adequately capture the specific needs of this population. Specific and valid measurement tools should be developed to identify the care needs of dying patients and their family caregivers. Some studies did not have high quality but were retained through group discussion, which may need to be taken into account when interpreting the results of this study.

\section{Implications for practice}

For healthcare professionals, it is vital to understand patients' and their family caregivers' needs comprehensively and provide them with tailored interventions to improve their satisfaction. Professionals, especially nurses, should enact patient demand-centred practice to overcome the challenges of organisation, education, emotions and communication to provide high-quality end-of-life care. More professional care resources and supportive care measures should be provided by the government for dying patients and their family caregivers so that patients receive high-quality end-of-life care.

\section{CONCLUSION}

In summary, findings of this meta-synthesis reveal that dying patients and their family caregivers in mainland China have many unmet needs. Dying patients want better control of pain and symptoms, emotional and spiritual care, professional care resources and to maintain daily life, maintain autonomy, die peacefully and in a dignified manner and prepare for death. Family members expressed their wish regarding receiving information about caregiving and disease, accessible professional care resources, initiative of diagnosis notification, respite care, social support and death management. It is imperative that these care needs should be focused on, and more resources should be provided for dying patients and their family caregivers. These findings highlight the importance of promoting palliative care in future.

\author{
Author affiliations \\ ${ }^{1}$ School of Nursing, Nanjing Medical University, Nanjing, Jiangsu, China \\ ${ }^{2}$ Emergency, Nantong First People's Hospital, Nantong, Jiangsu, China \\ ${ }^{3}$ China Jiliang University Hospital, Hangzhou, Zhejiang, China \\ ${ }^{4}$ Nanjing Medical University Library, Nanjing, Jiangsu, China \\ ${ }^{5}$ Jiangsu Vocational Institute of Commerce, Nanjing, Jiangsu, China \\ ${ }^{6}$ Department of Respiratory and Critical Care Medicine, Nanjing Medical University, \\ Nanjing, Jiangsu, China \\ ${ }^{7}$ Department of Respiratory and Critical Care Medicine, The First Affiliated Hospital \\ of Nanjing Medical University, Nanjing, Jiangsu, China \\ ${ }^{8}$ LKS Faculty of Medicine, University of Hong Kong, Hong Kong, China \\ ${ }^{9}$ School of Nursing, Peking University, Beijing, China \\ ${ }^{10}$ Evidence-Based Nursing: A Joanna Briggs Institute Centre of Excellence, Peking \\ University Health Science Centre, Beijing, China
}

Acknowledgements We thank LetPub (www.letpub.com) for its linguistic assistance during the preparation of this manuscript.

Contributors SZ contributed to the study conception and design, literature search, assessment, data extraction and analysis, manuscript writing and editing. $\mathrm{HZ}$ contributed to data extraction and analysis, manuscript writing and editing. XZ was responsible for the literature search and assessment. KL contributed to the study design, literature search and assessment. $X Y$ contributed to the contributed to the literature search. ZC contributed to the study conception, design and data extraction. CS was responsible for data analysis and manuscript editing. WX was responsible for data interpretation and manuscript revision. WL was responsible for updating the literature search. DP was responsible for JBI methodological guidance. YC, QX and HW were responsible for the study conception and design, manuscript drafting, and study supervision. SZ was responsible for the overall content as the guarantor. All the authors have reviewed the manuscript.

Funding 1. Project 'Studies on Construction of Core Competency Model and Development of Assessment Tool for Nurses of Hospice Care (72004099)' supported by NSFC. 2. Project 'The exploration of trajectories and intervention program of frailty for gastric cancer survivors based on the health ecology theory (82073407)' supported by NSFC. 3. Project 'Comparative study on hospice care mode between China and Canada (2017SJB0295)' supported by Philosophy and Social Science Foundation of universities in Jiangsu Province.

Competing interests None declared.

Patient consent for publication Not applicable.

Provenance and peer review Not commissioned; externally peer reviewed.

Data availability statement Data are available on reasonable request. The datasets used and analyzed during the current study are available from the corresponding author or the first author on reasonable request.

Supplemental material This content has been supplied by the author(s). It has not been vetted by BMJ Publishing Group Limited (BMJ) and may not have been peer-reviewed. Any opinions or recommendations discussed are solely those of the author(s) and are not endorsed by BMJ. BMJ disclaims all liability and responsibility arising from any reliance placed on the content. Where the content includes any translated material, BMJ does not warrant the accuracy and reliability of the translations (including but not limited to local regulations, clinical guidelines, terminology, drug names and drug dosages), and is not responsible for any error and/or omissions arising from translation and adaptation or otherwise.

Open access This is an open access article distributed in accordance with the Creative Commons Attribution Non Commercial (CC BY-NC 4.0) license, which permits others to distribute, remix, adapt, build upon this work non-commercially, and license their derivative works on different terms, provided the original work is properly cited, appropriate credit is given, any changes made indicated, and the use is non-commercial. See: http://creativecommons.org/licenses/by-nc/4.0/.

\section{ORCID iDs}

Hanfei Zhu http://orcid.org/0000-0001-5134-2287

Yan Cui http://orcid.org/0000-0002-0249-9883

\section{REFERENCES}

1 National Bureau of Statistics of China. China statistical Yearbook, 2019. Available: http://www.stats.gov.cn/tjsj/ndsj/2019/indexch.htm [Accessed $28 \mathrm{Mar} 2021]$.

2 National Cancer Center of China. The latest National cancer report in 2019. Available: https://www.sohu.com/a/296354370_707276 [Accessed 28 Mar 2021].

3 Sun JY, Hao XN, Bo T. Analysis of the status quo and needs of community care for disabled elderly in Beijing. Chin $J$ Health Policy 2016;9:57-64.

4 The Economist Intelligent Unit. 2015 quality of death index. Available: https://www.eiuperspectives.economist.com/sites/default/files/ images/2015\%20Quality\%20of\%20Death\%20Index\%20FINAL\% 20SC.pdf [Accessed 28 Mar 2021].

5 General Office of the State Council. Guiding opinions on promoting the combination of medical health and elderly services. Available: http://www.gov.cn/zhengce/content/2015-11/20/content_10328.htm [Accessed $28 \mathrm{Mar} 2021]$.

6 The State Council. The 13th five-year plan for healthy aging. Available: http://www.nhc.gov.cn/jtfzs/jslgf/201703/63ce9714 ca164840be76b362856a6c5f.shtml?tdsourcetag=s_pcqq_aiomsg [Accessed 28 Mar 2021].

7 National Health Commission of the People's Republic of China. Notice on the basic standards and management standards of hospice and palliative care centers (trial implementation). Available: http://www.nhc.gov.cn/cms-search/xxgk/getManuscriptXxgk.htm? id=2f50fdc62fa84cdd9d9a09d5162a661f [Accessed 28 Mar 2021]. 
8 Cui J, Song LJ, Zhou LJ, et al. Needs of family caregivers of advanced cancer patients: a survey in Shanghai of China. Eur $J$ Cancer Care 2014;23:562-9.

9 Molassiotis A, Yates P, Li Q, et al. Mapping unmet supportive care needs, quality-of-life perceptions and current symptoms in cancer survivors across the Asia-Pacific region: results from the International step study. Ann Oncol 2017;28:2552-8.

10 Li Q, Xu Y, Lin Y, et al. Psychometric properties of the Chinese version of the cancer survivors' unmet needs measure. Eur J Oncol Nurs 2020;46:101772.

11 Chung H, Harding R, Guo P. Palliative care in the greater China region: a systematic review of needs, models, and outcomes. J Pain Symptom Manage 2021;61:585-612.

12 Cheng G, Chen C. End-Of-Life needs of dying patients and their families in mainland China: a systematic review. Omega $2021 ; 30222821997340$

13 Aromataris E, Munn Z, eds. JBI manual for evidence synthesis. JBI, 2020. https://synthesismanual.jbi.global

14 Moher D, Liberati A, Tetzlaff J, et al. Preferred reporting items for systematic reviews and meta-analyses: the PRISMA statement. BMJ 2009;339:b2535.

15 Lockwood C, Munn Z, Porritt K. Qualitative research synthesis: methodological guidance for systematic reviewers utilizing metaaggregation. Int J Evid Based Healthc 2015;13:179-87.

16 Moola S, Munn Z, Tufanaru C. Chapter 7: Systematic reviews of etiology and risk. In: Aromataris E, Munn Z, eds. JBI manual for evidence synthesis. JBI, 2020. https://synthesismanual.jbi.global

17 Pluye P, Hong QN. Combining the power of stories and the power of numbers: mixed methods research and mixed studies reviews. Annu Rev Public Health 2014;35:29-45.

18 Wei CL, Fang Q, Yuan CR. Real experiences of nursing practice and demand of spiritual care in breast cancer patients receiving ora chemotherapy. Nurs J Chin PLA 2015;32:1-5.

$19 \mathrm{Xu} \mathrm{J}, \mathrm{WQ}$ Y, Jiang P. Qualitative study of current status and needs of caregivers of terminal cancer patient. Nurs J Chin PLA 2014;31:1-4.

20 Huang J. Study on the personnel construction and function of multidisciplinary team of cancer palliative care in Shanghai community. Available: https://kns.cnki.net/kcms/detail/detail.aspx? $\mathrm{dbcode}=$ CMFDanddbname $=$ CMFD2009andfilename $=2008154580$. nhandv=DdpTKPsqkMiH3kPDI8YNqESgqkYo59JqxgtGOeJKdxpy D3I6hjN206YaP2XSqyJJ [Accessed 18 Feb 2021]

21 Miao J, Cao WQ, Wang XY. Investigation on community care needs of patients with cancer and their family members in a community in Shanghai. Chin Nurs Res 2016;30:2386-90.

$22 \mathrm{ML} \mathrm{L}$, Luo ZQ. A qualitative study on needs for maintenance of dignity in patients with advanced cancer experiencing moderate to severe loss of dignity. J Nurs Sci 2019;34:79-82.

23 JY X, Dong FQ. Family members' needs for nursing services of terminal cancer patients: a qualitative research. Chin J Mod Nurs 2018;24:152-5.

24 HH W, Zhou N, Chen XY. Qualitative study of caregiver burden and needs in hospice care patients with advanced cancer. Chin Med Ethics 2019;32:1566-70.

25 Ji J, Yang YQ. Qualitative study on cancer patients' cognition to death and expection for hospice care. Chin J Mod Nurs 2016;22:4906-9.

26 Zhou SJ, Wen HM, Zhao QQ. Analysis of support needs of family caregivers of cancer patients receiving home - based palliative care. Chin Med Ethics 2020;33:1367-72.

27 WX L, Zeng CQ, OuYang XY. Quality of life and expectation of good death of cancer patients and their families. J Nurs 2018;25:71-4.

28 Cui X, Wang HL, CY X. Survey on the demands of main caregivers in patients with advanced liver cancer and analysis of its influencing factors. J Xinjiang Med Univ 2019;42:1211-5.

29 Liu JX, XY D, Dong JS. Investigation and countermeasure on the health education of family members' demands among Lung cancer patients. Mord Prev Med 2012;39:3039-40.
30 Zhao XJ, Yang Y, AP W. Investigation into need of hospice care among advanced cancer patients. J Nurs Sci 2015;30:27-30.

31 Cheng Q, Xu X, Liu X, et al. Spiritual needs and their associated factors among cancer patients in China: a cross-sectional study. Support Care Cancer 2018;26:3405-12.

32 Wang BL, Pang SQ, YL W. Caring needs of family members of elderly terminally-ill patients: a qualitative research. Nurs J Chin PLA 2016;33:11-16.

33 Wang T, Molassiotis A, Tan J-Y, et al. Prevalence and correlates of unmet palliative care needs in dyads of Chinese patients with advanced cancer and their informal caregivers: a cross-sectional survey. Support Care Cancer 2021;29:1683-98.

34 Liu X, Liu Z, Zheng R, et al. Exploring the needs and experiences of palliative home care from the perspectives of patients with advanced cancer in China: a qualitative study. Support Care Cancer 2021;29:4949-56.

35 Zalenski RJ, Raspa R. Maslow's hierarchy of needs: a framework for achieving human potential in hospice. J Palliat Med 2006;9:1120-7.

36 Zhang C, Xiao J, Yu Z, et al. Cancer pain management and the roles of pharmacists in China. Int J Clin Pharm 2021;43:383-5.

37 Yang B, Cui Z, Zhu X, et al. Clinical pain management by a multidisciplinary palliative care team: experience from a tertiary cancer center in China. Medicine 2020;99:e23312.

38 Chao C-SC, Chen C-H, Yen M. The essence of spirituality of terminally ill patients. J Nurs Res 2002;10:237-45.

39 Cheng QQ, Liang S, Chen YY. Spiritual needs among cancer patients: a review. Chin J Nurs 2016;51:330-5.

40 Ozen B, Ceyhan O, Büyükcelik A. Hope and perspective on death in patients with cancer. Death Stud 2020;44:412-8.

$41 \mathrm{Fu}$ YY, Chui EWT. Determinants of patterns of need for home and community-based care services among community-dwelling older people in urban China: the role of living arrangement and Filial Piety. $J$ App/ Gerontol 2020;39:712-21.

42 Nie X, Ye D, Wang Q, et al. Poor-Prognosis disclosure preference in cancer patient-caregiver dyads and its association with their quality of life and perceived stress: a cross-sectional survey in mainland China. Psychooncology 2016;25:1099-105.

43 Tang Y. Caregiver burden and bereavement among family caregivers who lost terminally ill cancer patients. Palliat Support Care 2019;17:515-22.

44 Vandepitte S, Van Den Noortgate N, Putman K, et al. Effectiveness of respite care in supporting informal caregivers of persons with dementia: a systematic review. Int $J$ Geriatr Psychiatry 2016;31:1277-88.

45 Ling $\mathrm{M}$, Wang $\mathrm{X}, \mathrm{Ma} \mathrm{Y}$, et al. A review of the current state of hospice care in China. Curr Oncol Rep 2020;22:99.

46 Woodman C, Baillie J, Sivell S. The preferences and perspectives of family caregivers towards place of care for their relatives at the endof-life. A systematic review and thematic synthesis of the qualitative evidence. BMJ Support Palliat Care 2016;6:418-29.

47 Maetens A, Beernaert K, De Schreye R, et al. Impact of palliative home care support on the quality and costs of care at the end of life: a population-level matched cohort study. BMJ Open 2019;9:e025180.

48 National Hospice and Palliative Care Organization. NHP-CO's facts and figures: hospice care in America. Available: https://www.nhpco. org/wp-content/uploads/NHPCO-Facts-Figures-2020-edition.pdf [Accessed 18 Feb 2021]

49 Chen SH, Xia HO, Lai XB. Identifying the barriers to the delivery of home-based end-of-life care in Shanghai:a concept mapping approach. J Nurs Sci 2021;36:72-6.

50 Zeng TY, QL L, ML W. Analysis on influencing factors of attitude toward illness condition informing of cancer patients. J Nurs Res 2009;23:670-2. 